\title{
Correlation between infectious disease and soil radiation in Japan: an exploratory study using national sentinel surveillance data
}

\author{
S. INAIDA ${ }^{1 *}$, T. TSUDA ${ }^{2}$ AND S. MATSUNO ${ }^{3}$ \\ ${ }^{1}$ Department of Epidemiology, Graduate School of Medicine, Dentistry and Pharmaceutical Sciences, Okayama \\ University, Okayama, Japan \\ ${ }^{2}$ Department of Human Ecology, Graduate School of Environmental and Life Sciences, Okayama University, \\ Okayama, Japan \\ ${ }^{3}$ Biomedical Science Association, Tokyo, Japan
}

Received 23 June 2016; Final revision 21 October 2016; Accepted 23 November 2016; first published online 16 January 2017

\section{SUMMARY}

We investigated the relationship between epidemics and soil radiation through an exploratory study using sentinel surveillance data (individuals aged $<20$ years) during the last three epidemic seasons of influenza and norovirus in Japan. We used a spatial analysis method of a geographical information system (GIS). We mapped the epidemic spreading patterns from sentinel incidence rates. We calculated the average soil radiation $[d m(\mu \mathrm{Gy} / \mathrm{h})]$ for each sentinel site using data on uranium, thorium, and potassium oxide in the soil and examined the incidence rate in units of $0.01 \mu \mathrm{Gy} / \mathrm{h}$. The correlations between the incidence rate and the average soil radiation were assessed. Epidemic clusters of influenza and norovirus infections were observed in areas with relatively high radiation exposure. A positive correlation was detected between the average incidence rate and radiation dose, at $r=0.61-0.84(P<0 \cdot 01)$ for influenza infections and $r=0.61-0.72(P<0.01)$ for norovirus infections. An increase in the incidence rate was found between areas with radiation exposure of $0<d m<0.01$ and $0.15 \leqslant d m<0 \cdot 16$, at $1.80[95 \%$ confidence interval (CI) 1.47-2.12] times higher for influenza infection and 2.07 (95\% CI 1.53$2 \cdot 61$ ) times higher for norovirus infection. Our results suggest a potential association between decreased immunity and irradiation because of soil radiation. Further studies on immunity in these epidemic-prone areas are desirable.

Key words: Epidemic, geographical information system (GIS), influenza, national sentinel surveillance, norovirus, soil radiation.

\section{INTRODUCTION}

Both influenza and norovirus epidemics follow a seasonal trend: a susceptible cohort and an efficient

\footnotetext{
* Author for correspondence: Dr S. Inaida, 2-5-1, Shikata-cho, Kita-ku, Okayama City, Okayama, Japan.

(Email: inaida@med.niigata-u.ac.jp)

The results of this study were presented at the 89th Annual Conference of the Japanese Association of Infectious Diseases (Kyoto, Japan, April 2015).
}

means of virus transmission are necessary to cause an epidemic. Our earlier studies demonstrated that seasonal epidemics of influenza and norovirus have similar geographical diffusion over time [1-3]. The characteristics of the epidemics were spatially related: they were confined to relatively small areas, showed gradual variation from the core of the epidemic to the periphery, and generated epidemic clusters. In some areas, the epidemic clusters were not affected by demographic variables alone, such as population 
density or family size, which is thought to play a key role in propagating viral transmission. This suggests the possibility of other environmental factors with a sustained effect on immunity, such as soil radiation.

Soil radiation is a major cause of environmental radiation and is thought to have a destructive effect on the human immunocycle through internal irradiation $[4,5]$. Previous studies suggested that irradiation resulted in a variety of haematopoietic disorders, such as abnormality of immunoglobulin or T-cell populations, mutated human cell-related components such as interferon, and adverse effects on gene transcription and DNA, with potential damage caused to bone marrow and thymus [4-21]. These haematopoietic functions and systems consist of the immunity-generating components, hence one of the effects of irradiation is thought to be a reduced level of immunity. Such results were observed for residents who were exposed to low-dose background radiation from the soil and workers at nuclear power plants or other occupational sites with radiation exposure, as well as survivors from the atomic bombings in Hiroshima and Nagasaki. However, the effect of irradiation on health outcomes, including immune dysfunction, remains unclear [22-24].

In this study, we conducted an exploratory study on the immunity impact of soil radiation by examining the relationship between the epidemics of infectious diseases and soil radiation level in Japan. We investigated an increase in the incidence rate of influenza and norovirus infection, as a susceptibility or immunity index, and we compared these incidence rates with the irradiation level from soil radiation by calculating a correlation. This study was intended to generate hypotheses about the health effects of soil radiation. For matching the incidence rates and soil radiation data, we used spatial overlay analysis using the geographical information system (GIS, ArcGIS 9.3v ESRI). Data on the incidence of infectious diseases were sourced from the National Epidemiological Surveillance of Infectious Diseases (NESID) [1] and soil sample data of uranium $(\mathrm{U})$, thorium $(\mathrm{Th})$, and potassium oxide $\left(\mathrm{K}_{2} \mathrm{O}\right)$ used for estimating the gamma irradiation level [25] were obtained from the website of the Geological Society of Japan.

\section{METHODS}

\section{Sentinel surveillance data}

We assessed sentinel surveillance data on individuals aged $<20$ years from the NESID during the last three epidemic influenza (2006-2007, 2007-2008, 2009-2010) and norovirus (2006-2007, 2007-2008, 2008-2009) seasons, whose epidemics recur with a considerable number of cases every year. Of note, the 2006-2007 norovirus season, which was the largest epidemic of the disease in the history of national surveillance, has been attributed to the emergence of a new GII.4 variant $[2,3]$. In addition, the 2009-2010 influenza season was a pandemic season in Japan. These epidemic seasons were included in this study to observe the effect of the emergence of new virus variants. For influenza, the 2006-2007 epidemic season was caused mainly by type B and subtype $\mathrm{H} 3$, and the 2007-2008 season was caused mainly by subtype H1. The 2008-2009 season, was the same as the previous season and continued to have subtype $\mathrm{H} 1$ as the predominant epidemic virus type, and the 2009-2010 season that had a lower incidence of norovirus were not included [26, 27]. For norovirus, the 2007-2008 season was caused by a predominant GII.4 variant [28].

The number of sentinel sites included about 5000 small- to medium-sized clinics. Influenza data (on influenza-like illnesses) were gathered at 2000 adult clinics and 3000 paediatric clinics. Norovirus data (i.e. gastroenteritis cases) were collected at about 3000 small- to medium-sized paediatric clinics. Of note, 3000 of the sentinel site paediatric clinics monitored both influenza and norovirus cases, and as such, the majority of data pertained to children aged $<15$ years. The number of sentinel sites was based on the catchment population of the administrative sector to which the respective health centres belonged. Selection of sentinel sites is randomly decided by the local health centres. To indicate the population background for sentinel sites, the number of population aged $<20$ years was extracted for a $1-\mathrm{km}$ radius of each sentinel through GIS using 2005 national census data.

Each sentinel clinic reported weekly the number of influenza cases to the prefectural heath institutes through regional health centres. Diagnosis was made based on the presence of four major symptoms (high fever, sudden outbreak of illness, upper respiratory inflammation, and whole body symptoms such as general malaise), and a rapid influenza testing kit was also frequently used to confirm the diagnosis. Gastroenteritis cases were reported weekly from each sentinel clinic based on the symptoms of diarrhoea, vomiting, and acute abdominal pain. Although this surveillance includes other causes of gastroenteritis such as bacterial or other viral causes, norovirus epidemics were also monitored by laboratory confirmation. The 
laboratory testing is conducted for about $10 \%$ of all cases through reverse transcription-polymerase chain reaction (RT-PCR) testing (at the laboratories of the prefectural health institutes). All these data include both sporadic cases and outbreaks. The full flow of surveillance data and the reporting system have been described in previous studies [29]. The significant difference of sentinel data was examined according to the Friedman test, i.e. a non-parametric test comparing observed datasets over time and their $P$ value (at significance level $P<0 \cdot 01$ ) examines a null hypothesis that the sampled data are identical for the different times when the data were collected [30]. This test was conducted using SPSS v. 21 (IBM Corp., USA) for each of the three seasons.

\section{Incidence rate}

We determined the population standard size for each sentinel site by calculating the population per clinic using the total population and the total number of clinics at the prefectural level. This was calculated for adjusting the population base level per clinic in prefectures. These data were obtained from online open sources based on surveys of medical institutions conducted by the Ministry of Health, Labour, and Welfare [31]. Subsequently, the incidence rates for the total number of incident cases [1,3] reported by a sentinel site were calculated against the prefectural population standard (hereinafter, the 'incidence') and taken into account for each of the three seasons to give a relative epidemic level. The calculation of incidence for influenza was conducted for the period of epidemic defined by the threshold of more than one incidence case per sentinel. For norovirus, the period of epidemic is defined from the 36th and 52nd epidemiological weeks of gastroenteritis cases, based on the pathogenic detection over a decade [3]. Then correlations of the incidences between the seasons were assessed using SPSS.

\section{Correlation between incidence and soil radiation dose}

The Kriging (ordinary kriging) interpolation method available in GIS Spatial Analyst enabled conversion of point-based data to surface data, and the epidemic cluster was depicted using the incidence for each of the three seasons (at 5-km grid cell size). Feature of spread of incidence was compared to background gamma radiation calculated according to observations of three radioactive components in the soil samples: uranium (U), thorium (Th), and potassium oxide $\left(\mathrm{K}_{2} \mathrm{O}\right)$ whose data are available at the website of the Geological Society of Japan [32]. The soil samples were collected from over 3000 sites (between 1999 and 2003) and these elements were extracted with $0 \cdot 1 \mathrm{~N} \mathrm{HCl}$ from the soil samples. The corresponding data were accessed online at the website of the Geological Society of Japan. The absorbed dose of $d(\mu \mathrm{Gy} / \mathrm{h})$ was calculated using $D^{-3}=13.0 \quad \mathrm{CK}_{2} \mathrm{O}$ $(\%)+5.4 \mathrm{CU}(\mathrm{ppm})+2 \cdot 7 \mathrm{CTh}(\mathrm{ppm})$ to estimate the dose of gamma irradiation at a height of $1 \mathrm{~m}$ from the ground $[32,33]$ and was depicted on a map according to the interpolation (natural neighbour) method (at $1-\mathrm{km}$ grid cell size) available in GIS Spatial Analyst. Natural neighbour is the method used to geographically calculate a subset of obtained samples to a query point and then weights them to fill a value to interpolate and is often used to map distribution of soil parameters [34, 35].

The mean absorbed dose $d m$ was calculated from the interpolated soil radiation using a $1-\mathrm{km}$ radius for each sentinel site in GIS. The average of the incidence for each radiation dose in units of $0.01 \mu \mathrm{Gy} / \mathrm{h}$ for each of the three seasons was calculated. Then, the moving averages of the incidences (using seven incidence values, including three values above and below each dose, in units rounded to $0 \cdot 01 \mu \mathrm{Gy} / \mathrm{h}$ ) and associated $95 \%$ confidence intervals (CIs) were calculated between $0<d m \leqslant 0 \cdot 15 \mu \mathrm{Gy} / \mathrm{h}$; the correlations between these values and the radiation dose were then assessed for both diseases in each of the three seasons. These calculations were conducted with SPSS.

\section{RESULTS}

\section{Distribution of incidence and soil radiation}

The areas with higher soil radiation tended to be located in the west of the country (Fig. 1a). For both influenza and norovirus infections, the Friedman test revealed a significant difference in sentinel data $(P<$ $0 \cdot 001)$ in all observed seasons. The spread of the incidences for both influenza and norovirus infections exhibited a similar pattern over the three seasons (maps of the 2006-2007 influenza and norovirus seasons are shown in Fig. 1b,c), and the mean correlation coefficients between the first season and the following two seasons were significantly high for influenza $(0.75, P<0.01)$ and norovirus $(0.90, P<0.01)$ infections. In areas with relatively higher soil radiation, there was an increased likelihood of epidemic clusters (spots of 
(a)
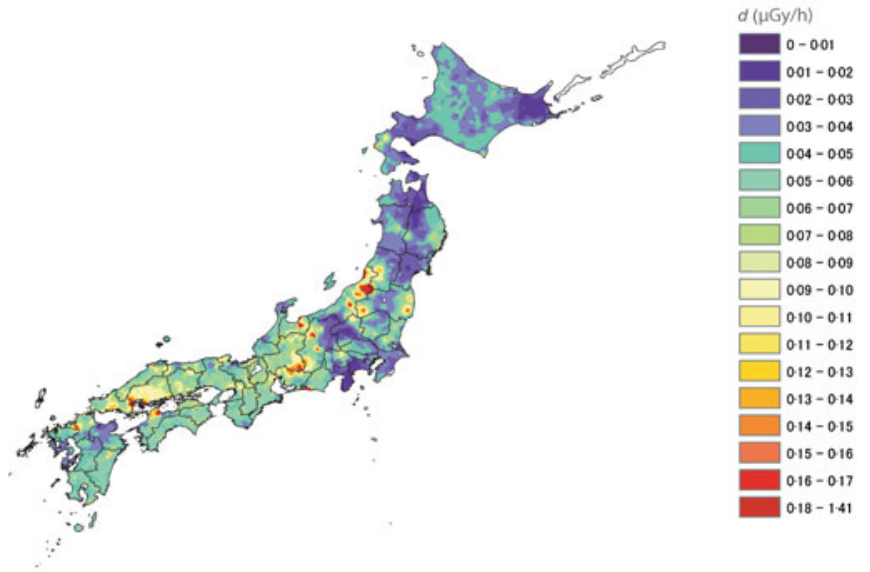

(b)

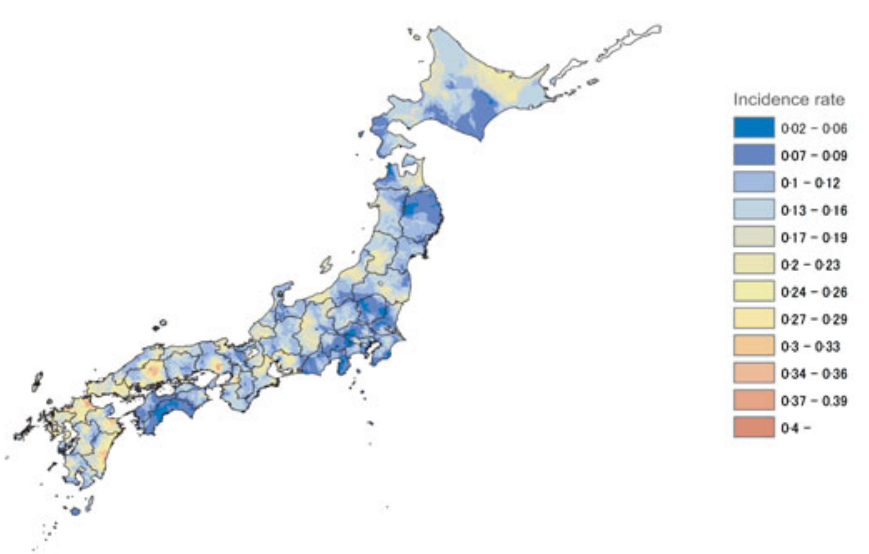

(c)

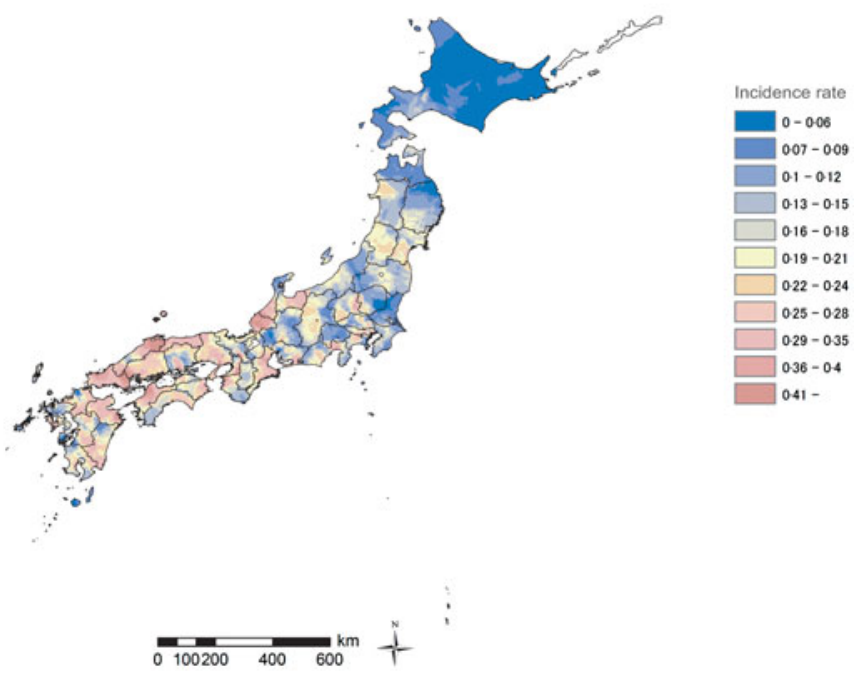

Fig. 1. Epidemic of infectious diseases and soil radiation in Japan. (a) Distribution of background gamma radiation $d(\mu \mathrm{Gy} / \mathrm{h}$ ) calculated from data of three radioactive components observed in soil samples (which were collected between 1999 and 2003), including uranium $(\mathrm{U})$, thorium $(\mathrm{Th})$, and potassium oxide $\left(\mathrm{K}_{2} \mathrm{O}\right)$, using $D^{-3}=13 \cdot 0 \mathrm{CK}_{2} \mathrm{O}(\%)+5 \cdot 4 \mathrm{CU}(\mathrm{ppm})+2 \cdot 7 \mathrm{CTh}(\mathrm{ppm})$ to estimate the dose at a height of $1 \mathrm{~m}$ from the ground. (b) Spread of incidence rate of influenza (2006-2007 season). (c) Spread of incidence rate of norovirus (2006-2007 season). Panels $(b)$ and $(c)$ were depicted using the incidence against the prefectural population standard size at each sentinel site. For panel $(c)$ the incidence rate of each sentinel site in the Kyushu area, where increased epidemic levels were observed compared to other areas, was adjusted (at minus $0 \cdot 1$ ) in order to provide an overview. 
Table 1. The distribution of the number of sentinel sites by dose of soil radiation and average of population aged $<20$ years (for 1-km radius of each sentinel site)

\begin{tabular}{lllll}
\hline \hline $\begin{array}{l}\text { Soil radiation } \\
d m(\mu \mathrm{Gy} / \mathrm{h})\end{array}$ & $\begin{array}{l}\text { Influenza (proportion } \\
\text { out of } 4496)\end{array}$ & $\begin{array}{l}\text { Average of } \\
\text { population }\end{array}$ & $\begin{array}{l}\text { Norovirus (proportion } \\
\text { out of } 3064)\end{array}$ & $\begin{array}{l}\text { Average of } \\
\text { population }\end{array}$ \\
\hline $0<d m<0 \cdot 01$ & $36(0 \cdot 008)$ & 904 & $27(0 \cdot 009)$ & 1464 \\
$0 \cdot 01 \leqslant d m<0 \cdot 02$ & $8(0 \cdot 002)$ & 1235 & $4(0 \cdot 001)$ & 1242 \\
$0 \cdot 02 \leqslant d m<0 \cdot 03$ & $142(0 \cdot 032)$ & 2148 & $94(0 \cdot 031)$ & 1427 \\
$0 \cdot 03 \leqslant d m<0 \cdot 04$ & $534(0 \cdot 115)$ & 3262 & $351(0 \cdot 115)$ & 1885 \\
$0 \cdot 04 \leqslant d m<0 \cdot 05$ & $923(0 \cdot 205)$ & 3453 & $616(0 \cdot 201)$ & 2087 \\
$0 \cdot 05 \leqslant d m<0 \cdot 06$ & $898(0 \cdot 200)$ & 2752 & $606(0 \cdot 198)$ & 1783 \\
$0 \cdot 06 \leqslant d m<0 \cdot 07$ & $632(0 \cdot 141)$ & 2047 & $414(0 \cdot 135)$ & 1363 \\
$0 \cdot 07 \leqslant d m<0 \cdot 08$ & $446(0 \cdot 099)$ & 2735 & $345(0 \cdot 113)$ & 1667 \\
$0 \cdot 08 \leqslant d m<0 \cdot 09$ & $316(0 \cdot 070)$ & 3582 & $227(0 \cdot 074)$ & 2322 \\
$0 \cdot 09 \leqslant d m<0 \cdot 10$ & $210(0 \cdot 047)$ & 3378 & $151(0 \cdot 049)$ & 1940 \\
$0 \cdot 10 \leqslant d m<0 \cdot 11$ & $122(0 \cdot 027)$ & 4362 & $77(0 \cdot 025)$ & 2349 \\
$0 \cdot 11 \leqslant d m<0 \cdot 12$ & $100(0 \cdot 022)$ & 4113 & $61(0 \cdot 020)$ & 2216 \\
$0 \cdot 12 \leqslant d m<0 \cdot 13$ & $56(0 \cdot 012)$ & 3324 & $41(0 \cdot 013)$ & 1959 \\
$0 \cdot 13 \leqslant d m<0 \cdot 14$ & $34(0 \cdot 008)$ & 2191 & $24(0 \cdot 008)$ & 1235 \\
$0 \cdot 14 \leqslant d m<0 \cdot 15$ & $22(0 \cdot 005)$ & 2378 & $15(0 \cdot 005)$ & 1608 \\
$0 \cdot 15 \leqslant d m<0 \cdot 16$ & $17(0 \cdot 004)$ & 2824 & $11(0 \cdot 004)$ & 1382 \\
Total & 4496 & - & 3064 & - \\
\hline \hline
\end{tabular}

Dose of soil radiation was calculated from data of three radioactive components observed in soil samples which were collected between 1999 and 2003 in Japan. A small number of sentinel sites located in areas with radiation exposure dose $0 \cdot 16 \leqslant d m$ $\mu \mathrm{Gy} / \mathrm{h}$, were excluded from this study.

Population (national census data of 2005) was extracted by 1-km radius for each sentinel site.

relatively higher incidence), although the number of incidences varied between different areas (Fig. 1).

Most sentinel sites for both influenza and norovirus were located in areas with exposure to the dose intervals of radiation of $0<d m \leqslant 0 \cdot 15 \mu \mathrm{Gy} / \mathrm{h}$, with the majority of sentinel sites having an exposure of $0<$ $d m<0.06 \mu \mathrm{Gy} / \mathrm{h}$ (Table 1). The population size (aged $<20$ years) within a $1-\mathrm{km}$ radius of each sentinel site showed relatively similar distribution over dose.

\section{Correlation between incidence and radiation exposure dose}

Across the analysed seasons, there was a gradual increase in the incidences associated with higher radiation doses (Table 2, Fig. 2). There were also some fluctuations in incidences. For example, the baseline incidence (which was the incidence at $0<d m<0.01$ $\mu \mathrm{Gy} / \mathrm{h}$ ) was higher in the 2009-2010 influenza season and the 2006-2007 norovirus season, which were both large outbreak seasons for each disease. With respect to the average incidences for the three seasons, the incidence for influenza infection was 1.80 (95\% CI $1 \cdot 47-2 \cdot 12)$ times higher and that for norovirus infection was $2.07(95 \%$ CI $1.53-2.61)$ times higher in areas with soil radiation doses of $0 \cdot 15 \leq d m<0 \cdot 16$ $\mu \mathrm{Gy} / \mathrm{h}$ compared to that in areas with the lowest doses of $0<d m<0.01 \mu \mathrm{Gy} / \mathrm{h}$.

Finally, the correlation between the fluctuation in the average of the incidences for influenza and norovirus infection and the soil radiation dose was significantly positive for all three seasons (Fig. 2, Table 3).

\section{DISCUSSION}

The distribution of higher incidences of epidemics of influenza and norovirus appeared to be geographically similar, and significantly positive correlations were found between the incidence of the first season and that of the following two seasons. The epidemic clusters tended to appear in areas with relatively higher soil radiation doses, and there was a positive correlation between incidence and soil radiation in all observed seasons. These results indicate a dosedependent effect of radiation on incidence and suggest that irradiation may potentially have an impact on immunity. Our data and study design are based on observations for geographically matching these viral infection rates and irradiation doses from the soil radiation across the country; there is also a limitation 

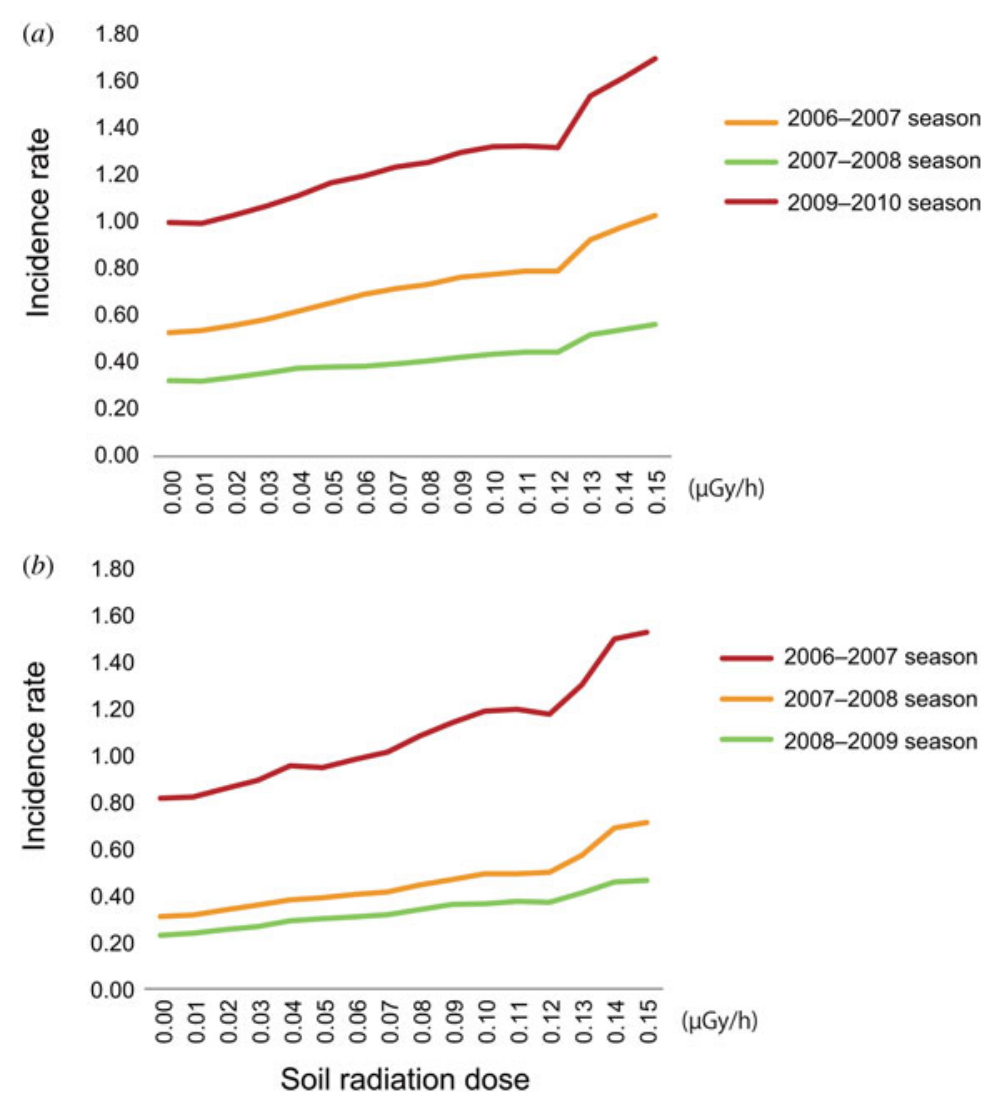

Fig. 2. Incidence rate and soil radiation dose in Japan. Changes in the moving averages of the incidence rates (calculated using seven values for the incidence rate, including three values above and below each dose, rounded to $0 \cdot 01 \mu \mathrm{Gy} / \mathrm{h}$ ) for each of the three epidemic seasons for influenza and norovirus (i.e. the fluctuation in the average of the incidence rate at $0.03 \mu \mathrm{Gy} / \mathrm{h}$ was calculated by taking the average of the incidence rate between $0 \mu \mathrm{Gy} / \mathrm{h}$ (rounded to $0<d m<0.005$ ) and $0.06 \mu \mathrm{Gy} / \mathrm{h}$ (rounded to $0.055 \leqslant d m<0.065$ ) based on the radiation dose data (calculated from data of three radioactive components observed in soil samples which were collected between 1999 and 2003) in Figure $1 a$ The value for the highest dose, $0 \cdot 15 \mu \mathrm{Gy} / \mathrm{h}$, was calculated by averaging the incidence rates between $0 \cdot 12$ and $0 \cdot 17 \mu \mathrm{Gy} / \mathrm{h}$ due to the limitation of obtaining data over $0.15 \mu \mathrm{Gy} / \mathrm{h}$. The data with $95 \%$ confidence intervals are shown in Table 2.

in consideration of other confounding circumstances. A further study is needed to consider these trends and the immunity impact of soil radiation.

Most epidemiological studies concerning the health effects of exposure to low-dose radiation have focused on cancer [36-39]. The few studies that have considered the immunity impact of soil radiation show that a mutation of the human interferon $\alpha-2 b$ gene accompanied with lower blood counts (i.e. white blood cells, mean corpuscular haemoglobin) was observed in individuals in irradiation areas with low-dose uranium, or in medical workers who were chronically exposed to radiation in Pakistan; this can be interpreted as a decrease in both anti-tumoural and antiviral immunity $[4,5]$. The other few studies that have considered infectious diseases in this context have reported an increase in the incidence of, or the death from infectious diseases, associated with low-dose radiation exposure [10-13, 40]. Some of other studies have also suggested an effect on immunity from haematopoietic disorders caused by irradiation $[6-9,15]$. The data framework and cohort were different in these studies, i.e. some were residents in higher dose areas and others were victims of the atomic bombs detonated in Hiroshima and Nagasaki, making it difficult to directly compare the results. However, they do provide a possible mechanism for the decline in immunity caused by exposure to lowdose radiation and, potentially, the subsequent increase in infections. Although it is likely that an immunity decrease can result in overall disease susceptibility, further studies are needed to consider immunity and susceptibility to these particular viral infections.

Soil radiation may cause internal contamination through the respiratory and digestive tracts via 
Table 2. Moving average of incidence rate (95\% confidence interval)

\begin{tabular}{|c|c|c|c|c|c|c|}
\hline \multirow[b]{2}{*}{ Soil radiation, $d m(\mu \mathrm{Gy} / \mathrm{h})$} & \multicolumn{3}{|l|}{ Influenza } & \multicolumn{3}{|l|}{ Norovirus } \\
\hline & 2006-2007 season & 2007-2008 season & 2009-2010 season & 2006-2007 season & 2007-2008 season & 2008-2009 season \\
\hline $0<d m<0.01$ & $0.53(0.45-0.61)$ & $0 \cdot 32(0 \cdot 28-0 \cdot 36)$ & $1 \cdot 00(0 \cdot 78-1 \cdot 23)$ & $0 \cdot 82(0 \cdot 49-1 \cdot 15)$ & $0 \cdot 31(0 \cdot 25-0 \cdot 37)$ & $0 \cdot 23(0 \cdot 14-0 \cdot 32)$ \\
\hline $0.01 \leqslant d m<0.02$ & $0.54(0.48-0.60)$ & $0 \cdot 32(0 \cdot 29-0 \cdot 35)$ & $1 \cdot 00(0 \cdot 85-1 \cdot 15)$ & $0 \cdot 83(0 \cdot 60-1 \cdot 05)$ & $0 \cdot 32(0 \cdot 27-0 \cdot 36)$ & $0 \cdot 24(0 \cdot 17-0 \cdot 30)$ \\
\hline $0.02 \leqslant d m<0.03$ & $0.56(0.49-0.63)$ & $0 \cdot 34(0 \cdot 29-0 \cdot 39)$ & $1 \cdot 03(0 \cdot 89-1 \cdot 18)$ & $0 \cdot 86(0 \cdot 67-1 \cdot 05)$ & $0 \cdot 34(0 \cdot 27-0 \cdot 41)$ & $0 \cdot 25(0 \cdot 19-0 \cdot 32)$ \\
\hline $0.03 \leqslant d m<0.04$ & $0.59(0.50-0.68)$ & $0 \cdot 36(0 \cdot 30-0 \cdot 42)$ & $1 \cdot 07(0 \cdot 92-1 \cdot 22)$ & $0 \cdot 90(0 \cdot 72-1 \cdot 07)$ & $0 \cdot 36(0 \cdot 29-0 \cdot 43)$ & $0 \cdot 27(0 \cdot 21-0 \cdot 33)$ \\
\hline $0 \cdot 04 \leqslant d m<0 \cdot 05$ & $0.62(0.51-0.73)$ & $0 \cdot 38(0 \cdot 31-0 \cdot 44)$ & $1 \cdot 12(0 \cdot 90-1 \cdot 33)$ & $0.96(0 \cdot 84-1 \cdot 08)$ & $0 \cdot 38(0 \cdot 32-0 \cdot 45)$ & $0 \cdot 29(0 \cdot 26-0 \cdot 33)$ \\
\hline $0 \cdot 05 \leqslant d m<0.06$ & $0.66(0.57-0.75)$ & $0 \cdot 38(0 \cdot 32-0 \cdot 44)$ & $1 \cdot 17(0 \cdot 99-1 \cdot 36)$ & $0.95(0.84-1 \cdot 07)$ & $0 \cdot 39(0 \cdot 33-0.45)$ & $0 \cdot 30(0 \cdot 27-0 \cdot 33)$ \\
\hline $0.06 \leqslant d m<0.07$ & $0.69(0.61-0.77)$ & $0 \cdot 39(0 \cdot 33-0 \cdot 45)$ & $1 \cdot 20(1 \cdot 03-1 \cdot 37)$ & $0.99(0 \cdot 90-1 \cdot 08)$ & $0 \cdot 41(0 \cdot 36-0 \cdot 45)$ & $0 \cdot 31(0 \cdot 28-0 \cdot 34)$ \\
\hline $0.07 \leqslant d m<0.08$ & $0.72(0.65-0.79)$ & $0 \cdot 40(0 \cdot 34-0 \cdot 45)$ & $1 \cdot 24(1 \cdot 10-1 \cdot 38)$ & $1 \cdot 02(0 \cdot 94-1 \cdot 10)$ & $0.42(0.37-0.46)$ & $0 \cdot 32(0 \cdot 30-0 \cdot 34)$ \\
\hline $0 \cdot 08 \leqslant d m<0.09$ & $0.74(0.69-0.78)$ & $0 \cdot 41(0 \cdot 37-0 \cdot 45)$ & $1 \cdot 26(1 \cdot 14-1 \cdot 37)$ & $1.09(0.98-1 \cdot 19)$ & $0.45(0.39-0 \cdot 50)$ & $0 \cdot 34(0 \cdot 30-0 \cdot 38)$ \\
\hline $0 \cdot 09 \leqslant d m<0.10$ & $0.77(0.71-0.83)$ & $0.43(0.36-0.49)$ & $1 \cdot 30(1 \cdot 16-1 \cdot 44)$ & $1 \cdot 15(0 \cdot 98-1 \cdot 31)$ & $0.47(0.39-0.55)$ & $0 \cdot 36(0 \cdot 30-0 \cdot 43)$ \\
\hline $0 \cdot 10 \leqslant d m<0 \cdot 11$ & $0 \cdot 78(0.72-0.84)$ & $0 \cdot 44(0.36-0 \cdot 52)$ & $1 \cdot 33(1 \cdot 17-1 \cdot 48)$ & $1 \cdot 20(1 \cdot 00-1 \cdot 39)$ & $0.49(0.39-0.60)$ & $0 \cdot 37(0 \cdot 30-0 \cdot 43)$ \\
\hline $0 \cdot 11 \leqslant d m<0 \cdot 12$ & $0.79(0.72-0.87)$ & $0.45(0.36-0.53)$ & $1 \cdot 33(1 \cdot 17-1 \cdot 49)$ & $1 \cdot 20(1 \cdot 02-1 \cdot 39)$ & $0.50(0.39-0.60)$ & $0 \cdot 38(0 \cdot 32-0.44)$ \\
\hline $0 \cdot 12 \leqslant d m<0 \cdot 13$ & $0.79(0.72-0.87)$ & $0.45(0.36-0.53)$ & $1 \cdot 32(1 \cdot 16-1 \cdot 49)$ & $1 \cdot 18(0 \cdot 97-1 \cdot 40)$ & $0 \cdot 50(0.41-0.60)$ & $0 \cdot 37(0 \cdot 31-0 \cdot 44)$ \\
\hline $0 \cdot 13 \leqslant d m<0 \cdot 14$ & $0.93(0.59-1.26)$ & $0.52(0.35-0.69)$ & $1 \cdot 54(1.04-2.05)$ & $1 \cdot 31(0.99-1.63)$ & $0.58(0.41-0.74)$ & $0.41(0 \cdot 31-0 \cdot 51)$ \\
\hline $0 \cdot 14 \leqslant d m<0 \cdot 15$ & $0.98(0.65-1 \cdot 32)$ & $0.54(0.38-0.71)$ & $1 \cdot 62(1 \cdot 12-2 \cdot 12)$ & $1 \cdot 51(1 \cdot 01-2 \cdot 00)$ & $0.69(0.42-0.97)$ & $0.46(0 \cdot 33-0.58)$ \\
\hline $0 \cdot 15 \leqslant d m<0 \cdot 16$ & $1 \cdot 03(0 \cdot 64-1 \cdot 42)$ & $0.57(0.37-0.76)$ & $1 \cdot 70(1 \cdot 14-2 \cdot 26)$ & $1 \cdot 54(0 \cdot 93-2 \cdot 15)$ & $0 \cdot 72(0 \cdot 38-1 \cdot 05)$ & $0.47(0.31-0.62)$ \\
\hline
\end{tabular}


Table 3. Correlation between incidence rates for influenza and norovirus infection and soil radiation

\begin{tabular}{llll}
\hline \hline Season & $2006-2007$ & $2007-2008$ & $2009-2010$ \\
\hline Influenza & $0 \cdot 84^{* *}$ & $0 \cdot 61^{* *}$ & $0 \cdot 66^{* *}$ \\
\hline Season & $2006-2007$ & $2007-2008$ & $2008-2009$ \\
\hline Norovirus & $0 \cdot 61^{* *}$ & $0 \cdot 72^{* *}$ & $0 \cdot 71^{* *}$ \\
\hline \hline
\end{tabular}

** $P<0 \cdot 01$

Pearson's correlation coefficients are shown for the fluctuating averages of the incidence rates for each dose (rounded to units of $0 \cdot 01 \mu \mathrm{Gy} / \mathrm{h})$ of soil radiation $(<0 \cdot 15 \mu \mathrm{Gy} / \mathrm{h})$ in Japan.

airborne transmission [41]. Our results demonstrated fluctuations in incidences correlated with radiation dose, i.e. differences in the rate of increase in the analysed seasons, suggesting that the incidence may also be affected by virus type. In correlations between the first season and the following two seasons, higher coefficients were found for norovirus infections than for influenza infections. This suggests that there is a greater tendency for norovirus infection to geographically recur over time, and likewise more susceptibility remains for norovirus infections than for influenza infections. Moreover, between lower and higher soil radiation doses there was a larger increase in incidences of norovirus infection compared to influenza, thus suggesting a potentially stronger effect of radiation on susceptibility to norovirus infection. The reason for this is not clear. However, earlier studies have reported that the stomach and intestinal tract, where norovirus is thought to multiply, are particularly sensitive to radiation [42-44]. One study has suggested that irritable bowel syndrome in children living in a radiation-contaminated area in Ukraine is potentially due to the irradiation effect on the $\mathrm{T}$ lymphocyte population; however, this was not compared with the impact on other internal tracts, or other diseases [7]. In addition, people tended to remain susceptible to norovirus infection over time as acquired immunity to norovirus is short-term [45]; therefore, any effect on the increasing incidence could be more noticeable compared to influenza infections [46]. Either way, the increasing incidence could be a sign of decreased immunity, potentially from irradiation doses as low as $0 \cdot 15 \mu \mathrm{Gy} / \mathrm{h}$.

The length of exposure to radiation is known to be an important factor that affects immunity [20]. According to a previous study, the majority of norovirus cases consisted of children aged $0-4$ years [3]. This may imply that radiation exposure of any duration before age 4 years could affect immunity. The current data pertained mainly to patients aged $<15$ years; therefore, the data does not allow for possible assessment of longer term effects. The most of sentinel sites were located in areas with radiation doses of $<0.15 \mu \mathrm{Gy} / \mathrm{h}$, which equals 1.3 (mGy) a year in cases of sustained exposure to radiation for $24 \mathrm{~h}$ a day. Meanwhile, previous studies have indicated that the effects of radiation could vary by individuals, although the mechanism involved is not well understood [17, 19]. Long-term observation studies are needed to fully evaluate the effect of radiation on immunity and overall health [4-16, 22-24, 47, 48].

Only a small number of the sentinel sites included in our study recorded soil radiation exposure in doses $>0 \cdot 15 \mu \mathrm{Gy} / \mathrm{h}$. Thus, our study is limited in its ability to make analysis regarding epidemic trends beyond that dose. Further, the data used in this study excluded other environmental data as well as background radiation from other terrestrial sources of radiation.

Although population size alone did not seem to be an epidemic factor, a demographic composition that includes a younger cohort may affect the spread of an epidemic $[1,3]$. The current study included incidence in individuals aged $<20$ years and there was no irregular conjunction of population aged $<20$ years for higher irradiation dose areas, which could have introduced potential bias by increasing the incidence in such areas. However, further studies regarding the epidemic trend according to age and sex are warranted. Additionally, the total number of clinics serving the total population in each area could affect the incidence, while our study was limited to calculations at the prefectural level. Influenza testing kits were mostly used to diagnose influenza infections and RT-PCR assays were conducted for specimens for $10 \%$ of norovirus infections; however, as the data were collected voluntarily by the physicians at sentinel sites, there could be a diagnostic bias. In Japan, a similar medical service with a universal medical insurance system is provided across the country; however, in rural areas where there are fewer residents than in metropolitan areas, the residential area of patients attending the same clinic may be larger than the $1-\mathrm{km}$ radius that we have used to calculate soil radiation for each sentinel clinic.

Although epidemic prevalence can be compared between epidemic clusters and smaller epidemic areas, the higher incidences tended to be located in less populated areas. As expected, in less populated 
areas, the population size for each sentinel site, which is a statistical parameter to calculate prevalence, is smaller than that in the populated core areas. Thus, the epidemic prevalence, i.e. the rate of incidence in relation to the population size for each sentinel site, in those less populated areas will be greater than that in populated core areas. The examination of prevalence between each epidemic cluster area and smaller epidemic areas would be desirable in a further study. We conducted this study to generate hypotheses of the effect of soil radiation on immunity; as such, the results should be interpreted with caution, and more studies are needed to test these hypotheses. Other potential causative factors such as differences in medical services or socioeconomic settings over areas, or an ecological fallacy, also require examination.

Although data on epidemics of influenza and norovirus could potentially be affected by other confounding factors, such as type-specific susceptibility to the virus and vaccination status (for influenza), the infections appeared to be clustered in similar geographical areas, suggesting further studies on immunity and associated factors in these epidemic areas are warranted.

\section{ACKNOWLEDGEMENTS}

This research received no specific grant from any funding agency, commercial or not-for-profit sectors.

\section{DECLARATION OF INTEREST}

None.

\section{REFERENCES}

1. Inaida S, et al. Geographic trends and spread of the pandemic (H1N1) 2009 in the metropolitan areas of Japan studied from the national sentinel data. Japanese Journal of Infectious Diseases 2011; 64: 473-481.

2. Inaida $\mathbf{S}$, et al. The south to north variation of norovirus epidemics from 2006-07 to 2008-09 in Japan. PLoS ONE 2013; 8: e71696.

3. Inaida $\mathbf{S}$, et al. The spatial diffusion of norovirus epidemics over three seasons in Tokyo. Epidemiology \& Infection 2014; 143: 522-528.

4. Shahid S, et al. Mutations of the human interferon alpha-2b (hIFN $\alpha-2 b)$ gene in low-dose natural terrestrial ionizing radiation exposed dwellers. Cytokine 2015; 76: 294-302.

5. Shahid S, et al. Mutations of the human interferon alpha-2b (hIFN- $\alpha 2$ b) gene in occupationally protracted low dose radiation exposed personnel. Cytokine 2015; 73: 181-189.
6. Amagi T, et al. Dysfunction of irradiated thymus for the development of helper T cells. Journal of Immunology 1987; 139: 358-364.

7. Sajjadieh MRS, et al. Affects of ionizing radiation on T-cell population lymphocyte: a risk factor of irritable bowel syndrome. Toxicology and Industrial Health 2010; 6: 323-330.

8. Godekmerdan A, et al. Diminished cellular and humoral immunity in workers occupationally exposed to low levels of ionizing radiation. Archives of Medical Research 2004; 35: 324-328.

9. Sajjadieh MRS, et al. Effect of ionizing radiation on development process of T-cell population lymphocytes in Chernobyl children. Iranian Journal of Radiation Research 2009; 7: 127-133.

10. Stewart AM, et al. Non-cancer effects of exposure to A-bomb radiation. Journal of Epidemiology \& Community Health 1984; 38: 108-112.

11. Stewart AM. Delayed effects of A-bomb radiation: a review of recent mortality rates and risk estimates for five-year Survivors. Journal of Epidemiology \& Community Health 1982; 36: 80-86.

12. Stewart AM, et al. A-bomb survivors: factors that may lead to a re-assessment of the radiation hazard. International Journal of Epidemiology 2000; 29: 708-714.

13. Stewart AM, et al. Radiation and marrow damage. British Medical Journal (Clinical Research Edition) 1982; 284: 1192.

14. Ohkita T. Acute effects. Journal of Radiation Research: 1975; 16 (Suppl. 1): 49-66.

15. Kusunoki Y, et al. Long-lasting alterations of the immune system by ionizing radiation exposure: implications for disease development among atomic bomb survivors. International Journal of Radiation Biology 2008; 84: 1-14.

16. Wuttke K, et al. Radiation induced micronuclei in subpopulations of human lymphocytes. Mutation Research 1993; 2: 181-188.

17. Bauman A, et al. The impact of natural radioactivity from a coal-fired power plant. Science of the Total Environment 1981; 17: 75-81.

18. Hagelstrom AH, et al. Chromosomal damage in workers occupationally exposed to chronic low level ionizing radiation. Toxicology Letters 1995; 76: 113-117.

19. Pohl-Rüling J. Low level dose induced chromosome aberrations in human blood lymphocytes. Radiation Protection Dosimetry 2014; 159: 10-19.

20. Gricienè B, et al. Cytogenetic monitoring of nuclear workers occupationally exposed to ionising radiation. Radiation Protection Dosimetry 1992; 1-4: 623-627.

21. Jahns J, et al. Influence of low dose irradiation on differentiation, maturation and T-cell activation of human dendritic cells. Mutation Research 2011; 709-710: 32-39.

22. McMahon DM, et al. Effects of long-term low-level radiation exposure after the Chernobyl catastrophe on immunoglobulins in children residing in contaminated areas: prospective and cross-sectional studies. Environmental Health 2014; 000: 13-36.

23. Oskouii MR, et al. Assessment of humoral immunity in workers occupationally exposed to low levels of ionizing radiation. Life Science Journal 2013; 5s. 
24. Daniak N, et al. Hematologic consequences of exposure to ionizing radiation. Experimental Hematology 2002; 30: $513-528$.

25. Beck HL, et al. In-situ $\mathrm{Ge}(\mathrm{Li})$ and $\mathrm{NaI}(\mathrm{Tl})$ gamma ray spectrometry. Health and Safety Laboratory AEC, Report HASL, 1972, pp. 258.

26. Infectious Agents Surveillance Report (IASR). Infectious Surveillance Centre, NIID, Japan (http://idsc.nih.go.jp/ iasr/prompt/graph-ke.html).

27. Inaida S, et al. Delayed norovirus epidemic in the 20092010 season in Japan: potential relationship with intensive hand sanitizer use for pandemic influenza. Epidemiology \& Infection 2016; 12: 2561-2567.

28. Motomura K, et al. Identification of monomorphic and divergent haplotypes in the 2006-2007 norovirus GII/4 epidemic population by genomewide tracing of evolutionary history. Journal of Virology 2008; 82: 11247-11262.

29. Taniguchi K, et al. Overview of infectious disease surveillance system in Japan, 1999-2005. Journal of Epidemiology 2007; 17 (Suppl: S3-1).

30. Michael R, et al. The use and interpretation of the Friedman test in the analysis of ordinal-scale data in repeated measures designs. Physiotherapy Research International 1996; 1: 221-228.

31. Ministry of Health, Labour and Welfare. Survey of medical institutions (http://www.mhlw.go.jp/english/database/db-hss/mi.html). Accessed 2 April 2015.

32. Geological Society of Japan. Radioactive elements in soil (2004) (https://gbank.gsj.jp/geochemmap/index_e. htm). Accessed 23 April 2014.

33. Minato S. Distribution of terrestrial $\gamma$ ray dose rates in Japan. Journal of Geography 2006; 1: 87-95.

34. Watson DF. Contouring: a Guide to the Analysis and Display of Spatial Data. Oxford: Elsevier, 1992, pp. 321.

35. Sarmah K, et al. Land suitability analysis for identification of summer paddy cultivation sites based on multi criteria evaluation through GIS. European Academic Research 2015; 2: 13584-13606.
36. Ujeno Y. Carcinogenetic hazard from natural background radiation in Japan. The Journal of Radiation Research 1978; 19: 205-212.

37. Land CE. Estimating cancer risks from low doses of ionizing radiation. Science 1980; 209: 1197-1203.

38. Rossi HH, et al. Radiation carcinogenesis at low doses. Science 1972; 175: 200-202.

39. Brenner DJ, et al. Cancer risks attributable to low doses of ionizing radiation: assessing what we really know. Proceedings of the National Academy of Sciences USA 2003; 100: 13761-13766.

40. Hodge FA, et al. Susceptibility to infection with Pasteurella tularensis and the immune response of mice exposed to continuous low dose rate gamma radiation. Journal of Infectious Diseases 1969; 120: 356-365.

41. Barcinski MA, et al. Cytogenetic investigation in a Brazilian population living in an area of high natural radioactivity. American Journal of Human Genetics 1975; 27: 802-806.

42. François A, et al. Inflammation and immunity in radiation damage to the gut mucosa. BioMed Research International 2013; 123241.

43. Somosy Z, et al. Morphological aspects of ionizing radiation response of small intestine. Micron 2002; 33: 167-178.

44. Coia LR, et al. Late effects of radiation therapy on the gastrointestinal tract. International Journal of Radiation Oncology, Biology, Physics 1995; 31: 1213-1236.

45. Siebenga JJ, et al. Epochal evolution of GGII.4 norovirus capsid proteins from 1995 to 2006. Journal of Virology 2007; 81: 9932-9941.

46. Reber AJ, et al. Seasonal influenza vaccination of children induces humoral and cell-mediated immunity beyond the current season: cross-reactivity with past and future strains. Journal of Infectious Diseases 2016; 214: 1477-1486.

47. Gloag D. Risks of low-level radiation - the evidence of epidemiology. British Medical Journal 1980; 281: 1479-1482.

48. Land CE. Uncertainty, low-dose extrapolation and the threshold hypothesis. Journal of Radiological Protection 2002; 3A: A129-135. 